\title{
Die automatische Kreatinbestimmung mit Hilfe der Diacetylreaktion
}

\author{
Von G. Gundlach, G. F. Hoppe-Seyler, H. JohanN ${ }^{1}$ ) \\ Aus der Klinisch-Chemischen Abteilung (Leiter: Priv.-Doz. Dr. G. Gundlach) der Urologischen Universitätsklinik \\ Homburg/Saar (Direktor: Prof. Dr. C. E. Alken)
}

(Eingegangen am 1. April 1968)

Eine empfindliche, reproduzierbare, automatische Methode zur Bestimmung von Kreatin in Serum, Harn und biologischen Flüssigkeiten wird auf der Grundlage der Diacetyl-Naphthol-Reaktion beschrieben. Die Durchführung der Reaktion bei niedrigen Temperaturen verhindert weitgehend die Umwandlung von Kreatinin zu Kreatin während der Reaktionszeit und ermöglicht die Bestimmung des Kreatins in Gegenwart großer Mengen an Kreatinin (z. B. bei 200fachem Uberschuß in Harn) ohne vorhergehende Abtrennung dieser Substanz. Der Einfluß anderer Störsubstanzen wird besprochen.

\section{The automatic determination of creatine with the aid of the diacetyl reaction}

A sensitive, reproducible, automatic method is described for the determination of creatine in serum, urine and biological fluids; it is based on the diacetyl-naphthol reaction. The reaction is performed at low temperatures, so that the conversion of creatinine into creatine during the reaction period is largely prevented. Thus creatine can be measured in the presence of large amounts of creatinine (e. g., a 200 fold excess in urine), without the preliminary separation of the two substances. The affect of other interfering substances is discussed.

Die analytischen Schwierigkeiten bei der Bestimmung des Kreatingehaltes in Serum und Urin haben immer wieder zu Verbesserungsvorschlägen der Bestimmungsmethoden geführt. Drei Verfahren haben sich in der Vergangenheit im wesentlichen zur Ermittlung des Kreatingehaltes biologischer Flüssigkeiten angeboten:

1. Umwandlung des Kreatins zu Kreatinin mit anschließender Bestimmung des Gesamtkreatinins meist mit alkalischer Pikrinsäure (1-3).

2. Direkte kolorimetrische Bestimmung der Methylguanidogruppe des Kreatins mit Diacetyl und $\alpha$-Naphthol (4-7).

3. Die enzymatische Umwandlung des Kreatins zu Kreatinphosphat und indirekte Messung der gebildeten Adenosindiphosphorsäure $(1,8)$.

Allen genannten Methoden haften Ungenauigkeiten an, die durch die mangelnde Spezifität der Farbreaktionen oder durch umständliche Verfahren bedingt sind. Besonders schwierig gestaltet sich der Nachweis von geringen Mengen Kreatin neben hohen Kreatininkonzentrationen, wie z. B. im Harn.

Die Analyse von Proben mit Hilfe des Diacetylreagenzes nach den herkömmlichen Methoden leidet - besonders bei der Untersuchung von Harn - unter der während der Farbbildung stetig erfolgenden Neubildung von Kreatin aus Kreatinin in der stark basischen Lösung. Durch Senkung der Reaktionstemperatur und Automatisierung des Verfahrens wird dieser. Störfaktor fast gänzlich ausgeschaltet.

\section{Material und Methoden}

\section{Reagenzien}

$\alpha$-Naphthol krist. reinst (Merck 6221), Butandion (2,3), 99-100\% (Schuchardt, München), Natriumcarbonat p. a. wasserfrei (Merck 6392), Natriumbydrogencarbonat p. a. (Merck 6329), Natriumchlorid p. a. (Merck 6404), Kreatin (Merck 5205); Serva chromato-

1) Teil der Dissertation $H$. Johann, Med. Fak. der Universität Homburg/Saar 1968. graphisch rein, Kreatinin (Merck 5206); Serva chromatographisch rein, alle anderen Substanzen waren, wenn nicht anders vermerkt, analysenrein.

\section{Lösungen}

1. 0,9 proz. Natriumchloridlösung $+1 \mathrm{~m} l$ einer $25 \mathrm{proz}$. Detergenslösung (Brij 35) pro Liter.

2. Butandion 1proz. in dest. Wasser. Direkt vor der Verwendung wird mit dest. Wasser 1:25 verdünnt.

3. $\alpha$-Naphthol-Lösung 8proz. in Natriumcarbonat-Natriumhydroxid-Puffer. Diese Lösung muß vor jedem Versuch frisch angesetzt werden. Sie ist dann mindestens für $3 \mathrm{Stdn}$. bei Raumtemperatur haltbar. Jedes Schütteln und Rühren während des Versuches ist zu vermeiden.

4. Natriumcarbonat-Natriumbydroxid-Puffer. $60 \mathrm{~g}$ Natriumhydroxid und $160 \mathrm{~g}$ Natriumcarbonat (wasserfrei) werden mit dest. Wasser zu $1 /$ gelöst. Die Lösung ist bei Raumtemperatur haltbar.

\section{Ausfübrung}

Die zu analysierende Probe wird mit der Natriumchloridlösung verdünnt einer Dialysatoreinheit zugeführt und gegen eine Natriumchloridlösung dialysiert. Das Dialysat wird auf $+7,5^{\circ}$ gekühlt, mit dem Diacetylreagenz versetzt und nach Durchlaufen einer Mischspirale weiter mit dem Naphtholreagenz zusammengebracht. Zur Farbentwicklung durchläuft dieses Gemisch eine etwa $8 \mathrm{~m}$ lange Glasspirale. Die Inkubationszeit der Reaktionslösung beträgt 9,3 Min. Die Farbtiefe wird bei $520 \mathrm{~nm}$ mit Hilfe

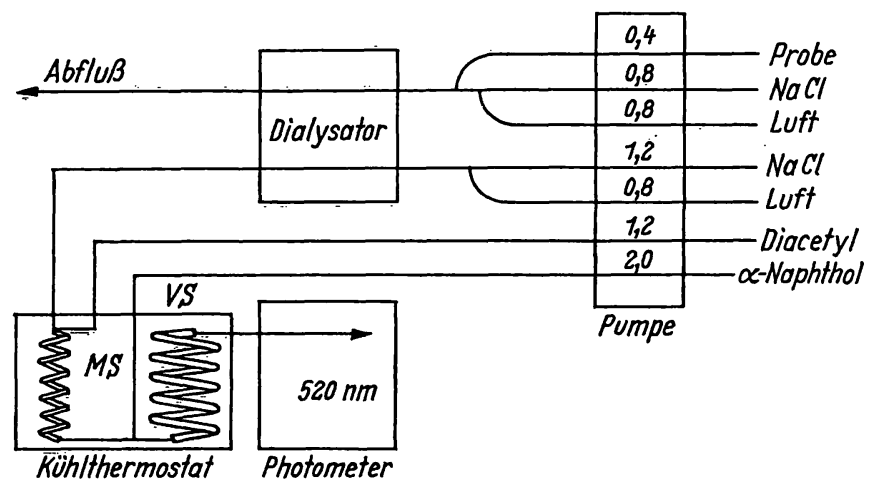

Abb. 1

Aufbau der Autoanalyzereinheit. Die Zahlenangaben bezeichnen die ml/Min. gefördertes Reagenz bz.w. Probe MS $=$ Mischspirale, VS $=$ Verzögerungsspirale 
eines Doppelstrahlkolorimeters registriert. Das Verhältnis der Reagenzien zueinander geht aus dem Flicßdiagramm in Abbildung 1 hervor. Die günstigste Probenfrequenz ergab sich bei 40 Proben/Std.

\section{Ergebnisse und Diskussion}

Die Automatisierung serienmäßig durchzuführender Bestimmungen wird heute in wachsendem Umfang notwendig. Aber auch bei einer kleineren Zahl zu untersuchender Proben kann die Automation sinnvoll werden. Die Konstanz z. B. der Parameter Zeit und Temperatur ist in der Automation besser als bei „Handmethoden“. Der Einfluß von Störsubstanzen und -faktoren kann dann u. U. vernachlässigt werden.

Die Diacetyl- $\alpha$-Naphtholreaktion zur quantitativen Kreatinbestimmung von BARRIT (4) in den Abwandlungen von Eggleton (5), RaAflaub (6), Kanig (7) wurde erstmals von GRIFFITH (9) automatisiert beschrieben. Diese Reaktion muß in alkalischer Lösung durchgeführt werden, wobei laufend Kreatinin in Kreatin überführt wird. GRIFFITH bestimmt daher in seinen auf Kreatin zu untersuchenden Proben gleichzeitig das vorhandene Kreatinin für den notwendigen Korrekturfaktor. Bei den „Handmethoden“ wird meist zweizeitig gemessen $(6,7)$.

Die oben beschriebene Methodik erlaubt die Bestimmung von 0-100 ppm Kreatin. Dabei ergibt sich eine lineare Abhängigkeit zwischen Extinkiion und Konzentration (Abb. 2). Die Empfindlichkeit der Methode be-

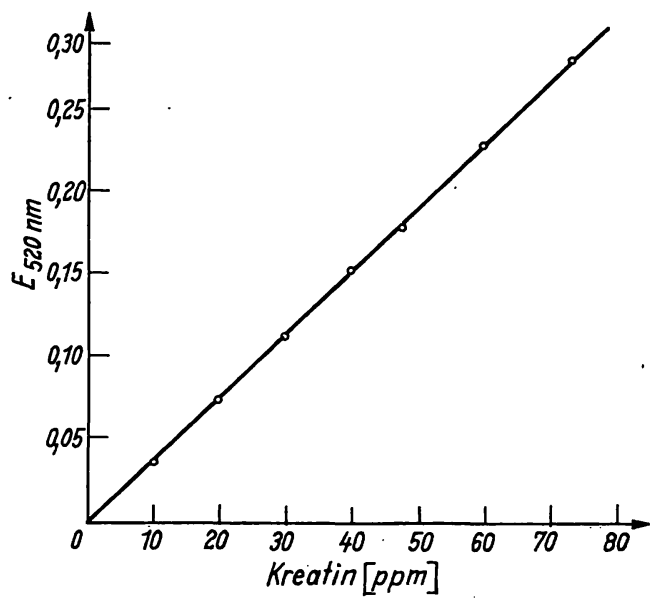

Abb. 2

Eichkurve für die Kreatinbestimmung

trägt $0,5 \mathrm{ppm}$. Bei einer Konzentration von $50 \mathrm{ppm}$ Kreatin beträgt der Variationskoeffizient $V=1,05 \%$, womit eine gute Reproduzierbarkeit in der Serie gegeben ist. Die Wiederfindungsquote in Harn und Serum beträgt $100 \%$, der Fehler liegt im Bereich des Variationskoeffizienten.

Der Einfluß unterschiedlicher Reagenzkonzentrationen auf die Farbausbeute geht aus Tabelle 1 hervor. Danach ist eine möglichst geringe Konzentration an Diacetyl für die Farbbildung am günstigsten. Bei der Verwendung von 20 ppm Diacetyl findet man jedoch bei höheren Kreatinkonzentrationen keine lineare Zunahme der
Tab. 1

Abhängigkeit der Farbbildung von Reagenzkonzentrationen

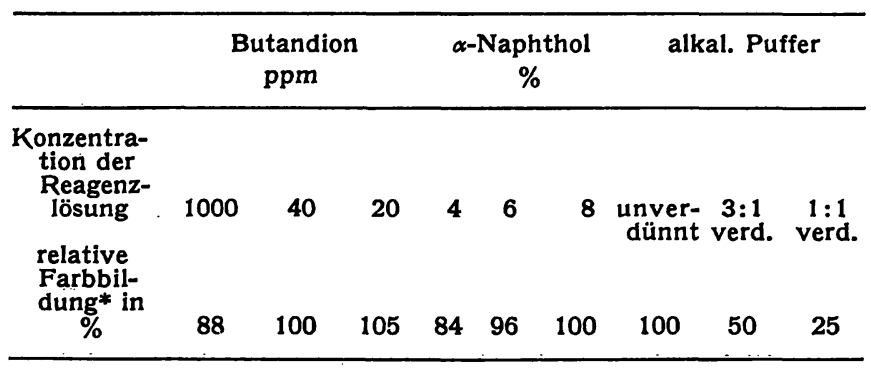

* Angegebene Zahlen beziehen sich auf die verwandte Standardkonzentration.

Extinktion. Eine zunehmende Konzentration an $\alpha$-Naphthol steigert die Farbausbeute. Höhere Konzentrationen als $8 \%$ verbieten sich wegen der Löslichkeit der Substanz bei z. B. $5^{\circ}$. Eine Senkung der Pufferkonzentration führt zu einer drastischen Verminderung der Farbintensität. Verdünnung des Puffers 1:1 mit dest. Wasser senkt die Farbbildung auf $25 \%$, verdünnt man 3:1, beträgt die Farbbildung etwa 50\%. Höhere Pufferkonzentrationen führen zur Ausfällung der Substanzen bei niederer Temperatur und beschleunigen die Umwandlung von Kreatinin zu Kreatin.

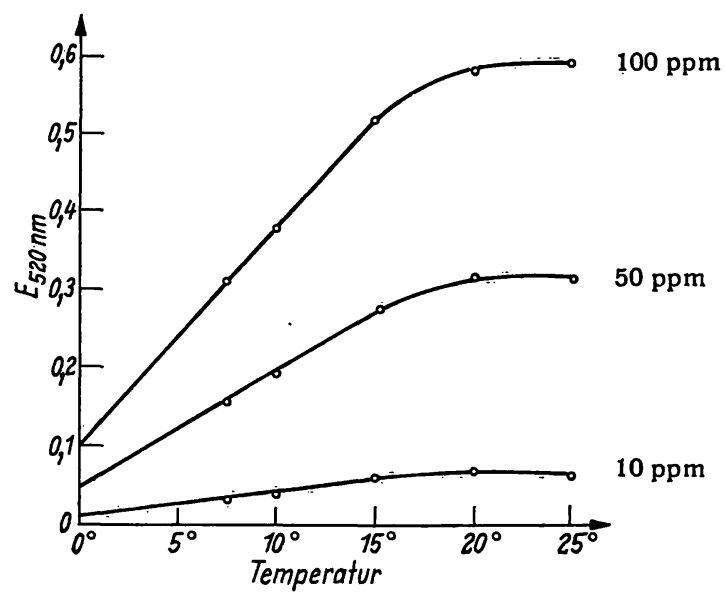

Abb. 3

Farbbildung des Kreatins in Abhängigkeit von dèr Temperatur bei konstanter Zeit

Die Bildung des Farbkomplexes ist bei konstanter Reaktionszeit abhängig von der Reaktionstemperatur. Aus Abbildung 3 geht hervor, daß unter den beschriebenen Standardbedingungen die Farbausbeute unter $15^{\circ}$ linear mit der Temperatur zunimmt.

Die Umwandlung von Kreatinin zu Kreatin nimmt jedoch erwartungṡgemäß (Abb. 4) mit steigender Temperatur exponentiell zu. Eine Senkung der Reaktionstemperatur führt $z$ war zu einer Verminderung der Farbausbeute, gleichzeitig aber zu einer überproportionalen Verringerung der Kreatinin-Kreatin-Umwandlung. Bei $7,5^{\circ}$ beträgt die Farbausbeute der Reaktion etwa $50 \%$ der bei Raumtemperatur zu erwartenden. An „scheinbarem Kreatin" aus der Kreatinin-Kreàtin-Umwandlung finden sich indessen, verglichen mit der Raumtemperatur, nur 9\%. Das bedeutet, daß in Gegenwart. von 
2000 ppm Kreatinin unter den von uns gewählten Bedingungen lediglich 1,5 ppm als "scheinbares"Kreatin" auftreten. Diese Konzentration führt auch in Gegenwart kleiner Mengen echten Kreatins, wie es im Harn zu finden ist, zu vernachlässigbaren Fehlern" (Abb. 5). Die für diese Untersuchungen herangezogenen Kreatininlösungen müssen stets frisch angesetzt werden.

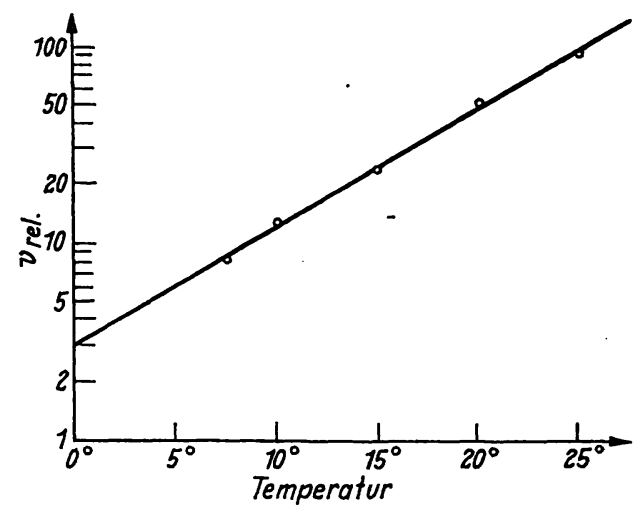

Abb. 4

Abhängigkeit der Umwandlung von Kreatinin zu Kreatin von der Temperatur unter den angegebenen Standardbedingungen. Die relative Umwandlungsgeschwindigkeit wurde logarithmisch aufgetragen

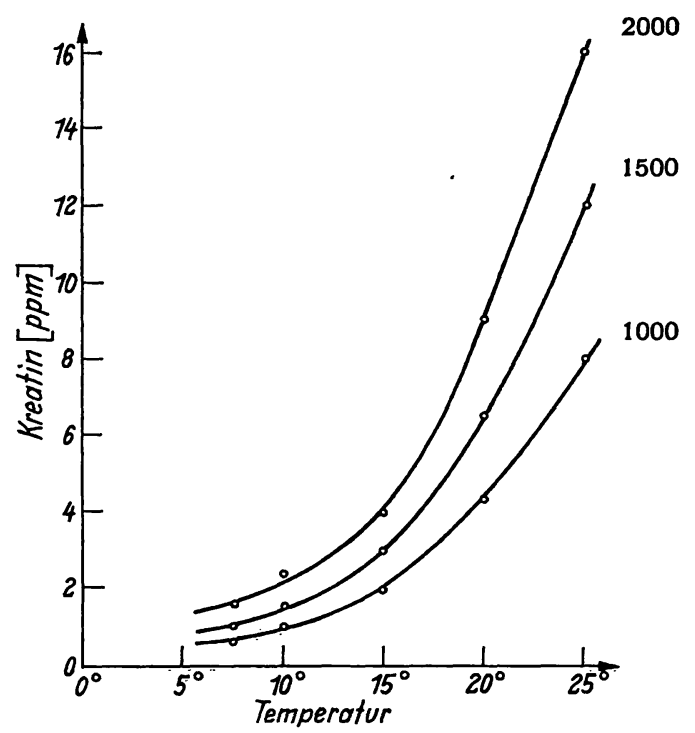

Abb. 5

Umwandlung von Kreatinin zu Kreatin in Abhängigkeit von der Umwandlung von Temperatur wahrend der Reaktion sich auf die Anfangskreatininkonzentration in $\mathrm{ppm}$.

Frische Lösungen enthalten weniger als $0,05 \%$ Kreatin, während eine bei $2^{\circ}$ drei Wochen lang aufbewahrte Lösung $0,5 \%$ Kreatin bezogen auf das Kreatinin enthält.

Wesentlich größer als der analytische Fehler ist die Umwandlung vorhandenen Kreatinins zu Kreatin in biologischen Flüssigkeiten bevor die Probe zur Analyse gelangt. Im Harn des erwachsenen Menschen findet sich eine mittlere Kreatininkonzentration von $1700 \mathrm{ppm}$ (10). Bereits in den harnableitenden Wegen und in der Blase kann sich die Umwandlung vollziehen. Abbildung 6 und Tabelle 2 geben eine Darstellung dieser Reaktion in Abhängigkeit von der Wasserstoffionenkonzentration.

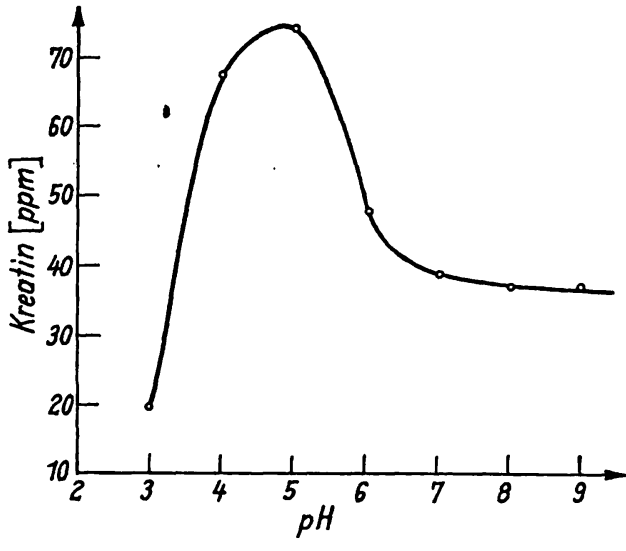

Abb. 6

Abhängigkeit der Umwandlung von Kreatinin in Kreatin vom pH. Bedingungen: 2000 ppm Kreatinin, 40, 24 Stdn.

Bei pH 5 durchläuft diese Reaktion eine Maximum. Da die $\mathrm{pH}$-Werte des Harnes normalerweise zwischen 5,5 und 7 schwanken, muß man mit Reaktionsgeschwindigkeiten rechnen, die um den Faktor von nahezu 2 im physiologischen Bereich unterschieden sind. Ein nächtlich produzierter, morgens ausgeschiedener Harn $(\mathrm{pH}$ 5 , mit einer angenommenen mittleren Verweildauer in der Blase von 6 Stdn.) kann somit die Konzentration von etwa $10 \mathrm{ppm}$ Kreatin vortäuschen. Es ist daher besonders wichtig, daß der frische Harn sofort gekühlt und angesäuert bis zur Analyse aufbewahrt wird. In einem 24-Stdn.-Harn finden sich dann höchstens weitere 5 ppm Kreatin. Auf den Tagesharn umgerechnet können also bis zu $15 \mathrm{mg}$ Kreatin nachgewiesen werden, die ursprünglich nicht vorhanden waren.

Tab. 2

Umwandlung von Kreatinin in Kreatin in Abhängigkeit von der Wasserstoffionenkonzentration. Die Ausgangslösung enthielt $2000 \mathrm{ppm}$ Kreatinin und hatte zur Einstellung des $\mathrm{pH}$ bereits bei Raumtemperatur gestanden

\begin{tabular}{|c|c|c|c|c|}
\hline \multirow[b]{2}{*}{ Żeit (Stdn.) } & \multirow[b]{2}{*}{$\mathrm{pH}$} & \multicolumn{3}{|c|}{ Kreatin (ppm) gefunden nach Inku- } \\
\hline & & $3^{\circ}$ & $22^{\circ}$ & $39^{\circ}$ \\
\hline 0 & $\begin{array}{l}3 \\
4 \\
5 \\
6 \\
7 \\
8 \\
9\end{array}$ & $\begin{array}{l}= \\
= \\
=\end{array}$ & $\begin{array}{l}2,0 \\
3,6 \\
2,8 \\
2,4 \\
2,0 \\
2,0 \\
2,0\end{array}$ & $\begin{array}{l}= \\
z \\
z\end{array}$ \\
\hline 3 & $\begin{array}{l}3 \\
4 \\
5 \\
6 \\
7 \\
8 \\
9\end{array}$ & $\begin{array}{l}2,6 \\
4,0 \\
4,4 \\
3,4 \\
2,5 \\
2,4 \\
2,4\end{array}$ & $\begin{array}{l}3,0 \\
5,0 \\
5,6 \\
4,4 \\
4,0 \\
3,8 \\
3,8\end{array}$ & $\begin{array}{l}4,4 \\
9,2 \\
9,8 \\
6,6 \\
6,4 \\
5,9 \\
6,0\end{array}$ \\
\hline 6 & $\begin{array}{l}3 \\
4 \\
5 \\
6 \\
7 \\
8 \\
9\end{array}$ & $\begin{array}{l}2,5 \\
3,6 \\
4,2 \\
3,2 \\
3,2 \\
3,0 \\
3,0\end{array}$ & $\begin{array}{l}3,0 \\
6,0 \\
6,8 \\
5,0 \\
4,4 \\
4,2 \\
4,2\end{array}$ & $\begin{array}{r}6,6 \\
16,8 \\
17,8 \\
11,8 \\
9,8 \\
9,8 \\
9,6\end{array}$ \\
\hline 12 & $\begin{array}{l}3 \\
4 \\
5 \\
6 \\
7 \\
8 \\
9\end{array}$ & $\begin{array}{l}2,2 \\
4,2 \\
4,4 \\
3,3 \\
2,8 \\
2,8 \\
2,9\end{array}$ & $\begin{array}{r}3,8 \\
8,6 \\
12,0 \\
6,4 \\
5,0 \\
5,0 \\
4,8\end{array}$ & $\begin{array}{l}11,0 \\
33,0 \\
35,0 \\
22,0 \\
18,0 \\
17,0 \\
17,0\end{array}$ \\
\hline 24 & $\begin{array}{l}3 \\
4 \\
5 \\
6 \\
7 \\
8 \\
9\end{array}$ & $\begin{array}{l}2,8 \\
5,6 \\
6,2 \\
4,4 \\
3,6 \\
3,7 \\
3,6\end{array}$ & $\begin{array}{r}6,4 \\
17,4 \\
19,4 \\
11,6 \\
9,4 \\
9,2 \\
9,2\end{array}$ & $\begin{array}{l}20,0 \\
67,5 \\
74,0 \\
47,5 \\
39,0 \\
37,5 \\
37,5\end{array}$ \\
\hline
\end{tabular}


Die alkalische Diacetylreaktion ist eine allgemeine Reaktion für Guanidine, bei denen mindestens eine freie Aminogruppe vorhanden sein muß (6). Der Einfluß von Arginin, Methylguanidin und Glycocyamin auf die Spezifität der Reaktion in bezug auf Kreatin wurde deshalb geprüft. Diese Substanzen sollen bei Raumtemperatur etwa $10 \%$ ihrer Konzentration an Kreatin vortäuschen (1). Durch Senkung der Reaktionstemperatur konnte die Spezifität der alkalischen Diacetylreaktion verbessert werden. Glycocyamin und Arginin täuschen weniger als 3,5\% der Farbausbeute gleicher Mengen an Kreatin vor. Durch den Einfluß dieser Substanzen werden also unter unseren Bedingungen und bei normaler Ausscheidung durch die Niere $(10,11)$ etwa nur $4 \mathrm{mg} / \mathrm{Tag}$ Kreatin zuviel gemessen (Tab. 3). Auf nicht mehr als $10 \mathrm{mg}$ steigt dieses "scheinbare Kreatin“ bei erhöhter Exkretion Guanidogruppen enthaltender Verbindungen z. B. bei der progressiven Muskeldystrophie an $(12,13)$. Bei der gleichzeitig stark vermehrten Kreatinausscheidung spielt diese Menge eine geringe Rolle. Selbst bei der enzymatischen Methode mit Kreatinphosphokinase wird Glycocyamin zu 2,5\% miterfaßt (1). Diese Methode benötigt außerdem viel Ausgangsmaterial und die Wiederfindungsquote liegt zwischen $86 \%$ und $110 \%$ (1). Einen Einfluß anderer potentieller Stör-
Tab. 3

Einfluß von Störsubstanzen auf die Kreatinbestimmung bei $7,5^{\circ}$

\begin{tabular}{|c|c|c|c|}
\hline Substanz & $\begin{array}{c}\text { Konzentration } \\
{[\mathrm{ppm}]}\end{array}$ & $\begin{array}{c}\text { scheinbare } \\
\text { Kreatin- } \\
\text { konzentration } \\
{[\mathrm{ppm}]}\end{array}$ & $\begin{array}{l}\text { Farbintensität } \\
\text { in \% der } \\
\text { Kreatin- } \\
\text { reaktion }\end{array}$ \\
\hline $\begin{array}{l}\text { Glycocyamin } \\
\text { Arginin } \\
\text { Methylguanidin } \\
\text { Harnstoff } \\
\text { Harnsäure* } \\
\text { Cystin } \\
\text { Andere Aminosäuren }\end{array}$ & $\begin{array}{r}200 \\
100 \\
1000 \\
20000 \\
500 \\
100 \\
100\end{array}$ & $\begin{array}{r}7 \\
3 \\
25 \\
0 \\
0 \\
0 \\
0\end{array}$ & $\begin{array}{l}3,5 \\
3,5 \\
2,5 \\
0 \\
0 \\
0 \\
0\end{array}$ \\
\hline
\end{tabular}

* Harnsäure allein senkt die Grundlinie. In Gegenwart von Kreatin $(20 \mathrm{ppm})$ ergibt sich keine meßbare Verminderung der Farbintensität.

substanzen wie Harnsäure, Harnstoff und verschiedener Aminosäuren (7) ließ sich in unserem Verfahren nicht nachweisen und der Harn mußte nicht - zur Ausschaltung von Störsubstanzen $(6,9)-$ verdünnt werden.

$\mathrm{Da}$ die Umwandlung des Kreatinins in den harnableitenden Wegen in der gleichen Größenordnung wie der durch Störsubstanzen bedingte Fehler liegt, scheint uns eine chromatographische $(14,15)$ oder elektrophoretische (16) Abtrennung des Kreatins, um die Genauigkeit zu erhöhen, nicht sinnvoll. Bei der Untersuchung von Serum treten die Störfaktoren und der Fehler, der durch das Kreatinin bedingt ist, nicht mehr hervor.

\title{
Literatur
}

1. Lauber, K., diese Z.. 3, 119 (1966). - 2. Dunicz, B. L., Clin. chim. Acta Amsterdam 9, 203 (1964). - 3. BENEDICT, S. R., J. biol. Chemistry 18, 191 (1914). - 4. Barritt, M. M., J. Pathol. Bacteriol. 42, 441 (1936). - 5. Eggleton, P.,'S. R. ElsDEN und N. Gough, Biochem. J. 37, 526 (1943). - 6. RAafLAuB, J. und I. Abelin, Biochem. Z. 321, 158 (1950). - 7. Kanig, K., HoppeSeyler's Z. physiol Chem. 306, 247 (1957). - 8. Berndt, E., H. U. Bergmeyer und H. MöLlering in H. U. Bergmeyer: Methoden der enzymatischen Analyse, S. 407, Verlag Chemie, Weinheim (1962). - 9. Griffith, W. J., Clin. chim. Acta (Amsterdam)
9, 213 (1964). - 10. Wissenschaftliche Tabellen Geigy 1960, 6. Auflage. - 11. ANDEs, J. E. und V. C. MYrers, J. Laborat. Clin. Med. S. Louis 22, 1447 (1937). - 12. Sullivan, M. X., W. C. Hess und F. Irreverre, J. biol. Chemistry 114, 633 (1936). 13. HobermanN, H. D., E. A. H. Sims und W. W. Engstrom, J. biol. Chemistry 173, III (1948). - 14. Paumgartner G., O. Kraupp und F. X. Fischer, Clin. chim. Acta (Amsterdam) 8, 960 (1963). - 15. RiNk, M. und D. KRebBer, J. Chromatogr. 21, 143 (1966). - 16. Fischl, J., S. Segal und Y. Yulzari, Clin. chim. Acta (Amsterdam) 10, 73 (1964).

Priv.-Doz. Dr. G. Gundlach 665 Homburg (Saar) Universitätskliniken

\section{Zur Eliminierung von Trübungsfehlern bei der Eiweißbestimmung mit der Biuretmethode}

\author{
Von Ch. Bode, H. Goebell und E. StähLER \\ Aus der Mediqinischen Universitätsklinik Marburg/Labn (Direktor: Prof. Dr. G. A. Martini)
}

(Eingegangen am 5. April 1968)

\begin{abstract}
1. Der blaue Biuretkomplex wird durch KCN-Zusatz komplett entfärbt. Diese Reaktion wird dazu benutzt, Lipidtrübungen in Eiweißlösungen zu erfassen und Fehler zu eliminieren. Die Methode ist einfach, wenig zeitaufwendig und liefert gut reproduzierbare Ergebnisse. 2. Störende Farbstoffbeimengungen (Hämoglobin) können mit der Methode ebenfalls ausgeschaltet werden.

3. Die Höhe der trübungsbedingten Fehler wird für Mitochondrien verschiedener Organe und menschliches Serum mit unterschiedlichem Lipidgehalt bestimmt.
\end{abstract}

